\title{
Finite Maxwell field and electric displacement Hamiltonians derived from a current dependent Lagrangian
}

\author{
M. Sprik \\ Department of Chemistry, University of Cambridge, Lensfield Road, Cambridge CB2 1EW, \\ United Kingdom
}

\section{ARTICLE HISTORY}

Compiled December 18, 2017

\begin{abstract}
In the common Ewald summation technique for the evaluation of electrostatic forces, the average electric field $\mathbf{E}$ is strictly zero. Finite uniform $\mathbf{E}$ can be accounted for by adding it as a new degree of freedom in an extended Lagrangian. Representing the uniform polarization $\mathbf{P}$ as the time integral of the internal current and $\mathbf{E}$ as the time derivative of an uniform vector field $\mathbf{A}$ we define such an extended Lagrangian coupling $\mathbf{A}$ to the total current $\mathbf{j}_{t}$ (internal plus external) and hence derive a Hamiltonian resembling the minimal coupling Hamiltonian of electrodynamics. Next applying a procedure borrowed from nonrelativistic molecular electrodynamics the $\mathbf{j}_{t} \cdot \mathbf{A}$ coupling is transformed to $\mathbf{P} \cdot \mathbf{D}$ form where $\mathbf{D}$ is the electric displacement acting as an electrostatic boundary condition. The resulting Hamiltonian is identical to the constant-D Hamiltonian obtained by Stengel, Spaldin and Vanderbilt (SSV) using thermodynamic arguments (Nature Physics 5, 304 (2009)). The corresponding SSV constant-E Hamiltonian is derived from an alternative extended Lagrangian.
\end{abstract}

\section{KEYWORDS}

Molecular dynamics, polarization, finite electric fields, extended Lagrangians.

\section{Introduction}

In their seminal 2009 paper Stengel, Spaldin and Vanderbilt (SSV)[1] presented two finite field Hamiltonians, one for constant macroscopic Maxwell field $\mathbf{E}$ and one for constant electric displacement field $\mathbf{D}$. In the formulation for a classical molecular dynamics (MD) system the constant-E Hamiltonian is written as

$$
H_{E}=\sum_{i}^{N} \frac{\mathbf{p}_{i}^{2}}{2 m_{i}}+V_{\mathrm{PBC}}\left(\mathbf{r}^{N}\right)-\frac{\Omega}{8 \pi} \mathbf{E}^{2}-\Omega \mathbf{E} \cdot \mathbf{P}
$$

and the constant-D Hamiltonian as

$$
H_{D}=\sum_{i}^{N} \frac{\mathbf{p}_{i}^{2}}{2 m_{i}}+V_{\mathrm{PBC}}\left(\mathbf{r}^{N}\right)+\frac{\Omega}{8 \pi}(\mathbf{D}-4 \pi \mathbf{P})^{2}
$$

Email: ms284@cam.ac.uk 
where $\mathbf{p}_{i}=m_{i} \mathbf{v}_{i}$ is the momentum of particle $i$ with position vector $\mathbf{r}_{i}$ and velocity $\mathbf{v}_{i}=\dot{\mathbf{r}}_{i}$. The mass of particle $i$ is $m_{i}$. The potential $V_{\mathrm{PBC}}\left(\mathbf{r}^{N}\right)$ is the potential energy of the $N$ particle system with the electrostatic interactions evaluated using standard Ewald summation (no surface term) $[2,3] . \Omega$ is the volume of the periodic supercell.

The electric field $\mathbf{E}$ in Eq. 1 is the uniform Maxwell field and is a fixed parameter. $\mathbf{P}$ is the uniform polarization and is a dynamical degree of freedom. While $\mathbf{E}$ acts as an applied field, it implicitly includes all screening interactions and is not to be confused with the bare external field $\mathbf{E}_{0}$. This property is specific to standard Ewald summation ("tin foil" boundary conditions) as already noted by Yeh and Berkowitz in their finite field MD study of liquid water[4].

True to the background in solid state physics, uniform polarization $\mathbf{P}$ in the SSV method is not based on a multipole expansion of molecular charge distributions but is defined in terms of the internal current $\mathbf{j}$

$$
\mathbf{j}=\frac{d \mathbf{P}}{d t}=\dot{\mathbf{P}}
$$

Conform the convention in the dielectric theory of insulators, the magnetisation $\mathbf{M}$ is set to zero[5,6]. For a classical system of point charges $\mathbf{j}$ is a simple direct sum of the contributions of the individual particles

$$
\mathbf{j}=\frac{1}{\Omega} \sum_{i}^{N} q_{i} \mathbf{v}_{i}
$$

where $q_{i}$ is the charge of particle $i$. Carrying out the time integral we recover an expression for polarization as a dipole density

$$
\mathbf{P}=\frac{1}{\Omega} \sum_{i}^{N} q_{i}\left(\mathbf{r}_{i}-\mathbf{r}_{i}(0)\right)
$$

referred, however, to the positions $\mathbf{r}_{i}(0)$ at $t=0$, the start of the time integration. $\mathbf{P}$ of Eq. 5 is the polarization generated by the (transient) flow of all charges in the system, including possible free (mobile) charge[7]. If a particle crosses the boundary of the MD cell it must be followed into the next cell, rather than reintroduced on the opposite side of the cell it left. $\mathbf{P}$ is identical to the itinerant polarization introduced by Caillol, Levesque and Weiss in their pioneering MD calculations of the electric response of aqueous ionic solutions (electrolytes)[3, 8-10] (See also Sega et al.[11]).

$\mathbf{D}$ in Eq. 2 is the dielectric displacement and is a fixed parameter. $\mathbf{D}$ is related to the Maxwell field and polarization as

$$
\mathbf{D}=\mathbf{E}+4 \pi \mathbf{P}
$$

Eq. 6 is the fundamental equation of the Maxwell theory of dielectric materials. It should be kept in mind, again, that in the SSV approach all charge in the system is accounted for by the polarization. With explicit external charge removed the key source of $\mathbf{D}$ is the charge on the "virtual" electrodes at infinity (the tin foil) acting as the electric boundary conditions for Ewald summation. For constant-D (Eq. 2) this charge is fixed (open circuit conditions) while for constant-E the tin foil charge fluctuates in time responding to the polarization $[1,12,13])$. This is distinctly different 
form the practice in the physical chemistry of ionic solutions where $\mathbf{D}$ is the electric field generated by the mobile ions treated as external charge (see the example of Refs. 8-11). This also implies that in the SSV picture $\Delta V=-E L$ can be directly interpreted as the voltage over the MD cell in the usual orthorombic geometry of the field E along a side with length $L$. As a result, the Hamiltonians Eq. 1 and 2 are perfectly adapted for applications to electrochemical model systems[7].

The constant field Hamiltonians Eqs. 1 and 2 were derived by Stengel, Spaldin and Vanderbilt on the basis of thermodynamic arguments $[1,12,13]$ following in essence Landau and Lifschitz[14]. $H_{E}$ and $H_{D}$ are however fully microscopic Hamiltonians and it should be possible to obtain these expressions from a Lagrangian using the formal arguments of theoretical mechanics. In this contribution we show that this can be achieved by using a simplified (non-radiative) form of non-relativistic molecular electrodynamics[15-21]. We are exploiting the extensive literature on transforming the velocity form (coupling of momentum to vector potential) to the length form (coupling of dipole moment to electric field) of the Hamiltonian of atoms and molecules in an electromagnetic magnetic field. This long and profound development was initiated by the classic paper of Power and Zienau[22]. The simplification for our system is that magnetic effects will be ignored. The challenge is to adapt this formalism intended for finite systems in vacuum to uniform fields under periodic boundary conditions. The formalism outlined below, treating the Maxwell field as a dynamical variable, has also some connection to the so called Maxwell equations molecular dynamics (MEMD) method developed by Maggs[23-26]. This concerns in particular the realization that uniform Maxwell fields and polarization require special treatment[27].

\section{Constant-D}

\subsection{Electrodynamics-like Lagrangian and Hamiltonian in $\mathrm{j} \cdot \mathrm{A}$ form}

A uniform electric field cannot be specified in terms of a charge density using Gauss law. Neither is it accounted for by Ewald summation in the standard form ("tin foil" boundary conditions). The uniform electric field under Ewald periodic boundary conditions will have to be treated as an additional dynamical degree of freedom $\mathbf{E}$ with an equation of motion derived from an extended Lagrangian. This requires defining a velocity for $\mathbf{E}$. The alternative suggested by electrodynamics[28] is to use the time integral $\mathbf{A}$ of $\mathbf{E}$ as the basic dynamical variable.

$$
\mathbf{E}=-\frac{d \mathbf{A}}{d t}=-\dot{\mathbf{A}}
$$

The sign is for reasons of consistency with the vector potential $\mathbf{A}(\mathbf{r})$ of electrodynamics[17, 28]. A of Eq. 7 is, however, by definition uniform. Hence

$$
\nabla \cdot \mathbf{A}=0, \quad \nabla \times \mathbf{A}=0
$$

which eliminates magnetic induction B. Substituted in Faradays's law this would imply

$$
\nabla \times \mathbf{E}=-\frac{1}{c} \frac{\partial \mathbf{B}}{\partial t}=0
$$


consistent with Eq. 7 with $c$ set to unity. In this quasistatic nonmagnetic limit the electrodynamical Lagrangian is simplified to

$$
L_{D}=\frac{1}{2} \sum_{i}^{N} m_{i} \mathbf{v}_{i}^{2}+\frac{\Omega}{8 \pi} \dot{\mathbf{A}}^{2}-V_{\mathrm{PBC}}\left(\mathbf{r}^{N}\right)+\Omega \mathbf{j}_{t} \cdot \mathbf{A}
$$

$\mathbf{j}_{t}$ is the total uniform current density consisting of a external (controlled) current $\mathbf{j}_{0}$ and the internal current $\mathbf{j}$ of Eq. 4

$$
\mathbf{j}_{t}=\mathbf{j}_{0}+\mathbf{j}
$$

As we will argue below, rather than corresponding to a flow of explicit external charge, $\mathbf{j}_{0}$ must be regarded as a displacement current.

The canonical momenta of the particles are obtained from $L_{D}$ of Eq. 10 as

$$
\mathbf{p}_{i}=\frac{\partial L_{D}}{\partial \mathbf{v}_{i}}=m_{i} \mathbf{v}_{i}+q_{i} \mathbf{A}
$$

The canonical momentum $\mathbf{p}_{i}$ of particle $i$ differs from its kinetic momentum $m_{i} \mathbf{v}_{i}$ similar to the minimal coupling scheme of electrodynamics. Substituting in the Lagrangian equations of motion.

$$
\frac{d}{d t} \frac{\partial L}{\partial \mathbf{v}_{i}}=\frac{\partial L}{\partial \mathbf{r}_{i}}
$$

we obtain

$$
m_{i} \dot{\mathbf{v}}_{i}+q_{i} \dot{\mathbf{A}}=-\frac{\partial V_{\mathrm{PBC}}}{\partial \mathbf{r}_{i}}
$$

Inserting Eq. 7 we recover the expected Newtonian equation of motion of a charged particle in an electric field

$$
m_{i} \dot{\mathbf{v}}_{i}=-\frac{\partial V_{\mathrm{PBC}}}{\partial \mathbf{r}_{i}}+q_{i} \mathbf{E}
$$

The $q_{i} \mathbf{E}$ term in Eq. 15 adds the force exerted by the uniform electric field which is missing from $V_{\mathrm{PBC}}$ consistent with the SSV interpretation of $\mathbf{E}$ as the Maxwell field.

To derive the equation of motion of $\mathbf{E}$ we first write down the canonical momentum $\boldsymbol{\Pi}_{A}$ of $\mathbf{A}$ as imposed by the Lagrangian Eq. 10

$$
\boldsymbol{\Pi}_{A}=\frac{\partial L_{D}}{\partial \dot{\mathbf{A}}}=\frac{\Omega}{4 \pi} \dot{\mathbf{A}}=-\frac{\Omega}{4 \pi} \mathbf{E}
$$

where in the second identity we have used Eq. 7. The Lagrangian equations of motion follow as usual by working out

$$
\frac{d}{d t} \frac{\partial L_{D}}{\partial \dot{\mathbf{A}}}=\frac{\partial L_{D}}{\partial \mathbf{A}}
$$


and we find

$$
\dot{\mathbf{E}}=-4 \pi \mathbf{j}_{t}
$$

Eq. 18 can be regarded as a "dielectric" (non-magnetic) limit of the Ampere-Maxwell equation as already pointed out by Maggs[27] (See also Hirst[5]). Substitution of Eqs. 11 and 3 separates the internal and external currents

$$
\dot{\mathbf{E}}=-4 \pi\left(\mathbf{j}_{0}+\dot{\mathbf{P}}\right)
$$

Eq. 19 together with Eq. 15 are a coupled set of equations of motion which determine the time evolution of the system, particles and uniform electric field. Comparing to the fundamental equation 6 of dielectric theory we are led to introduce a uniform electric displacement

$$
\dot{\mathbf{D}}=-4 \pi \mathbf{j}_{0}
$$

which can vary in time depending on the controlled time dependence of the external current $\mathbf{j}_{0}$. A discussion of the physical interpretation of Eq. 20 will be deferred to section 3 .

The Hamiltonian of the dynamics defined by Eq. 10 is obtained from the equation

$$
H_{D}=\sum_{i}^{N} \mathbf{p}_{i} \cdot \mathbf{v}_{i}+\Pi_{A} \cdot \dot{\mathbf{A}}-L_{D}
$$

Substituting Eqs. 10, 11, 12 and 16 yields

$$
\begin{aligned}
H_{D} & =\frac{1}{2} \sum_{i}^{N} m_{i} \mathbf{v}_{i}^{2}+\sum_{i} q_{i} \mathbf{v}_{i} \cdot \mathbf{A}+\frac{\Omega}{8 \pi} \dot{\mathbf{A}}^{2}+V_{\mathrm{PBC}}\left(\mathbf{r}^{N}\right)-\left(\Omega \mathbf{j}_{0}+\sum_{i}^{N} q_{i} \mathbf{v}_{i}\right) \cdot \mathbf{A} \\
& =\frac{1}{2} \sum_{i}^{N} m_{i} \mathbf{v}_{i}^{2}+\frac{\Omega}{8 \pi} \dot{\mathbf{A}}^{2}+V_{\mathrm{PBC}}\left(\mathbf{r}^{N}\right)-\Omega \mathbf{j}_{0} \cdot \mathbf{A}
\end{aligned}
$$

Reintroducing canonical momenta for the particles (Eq. 12) and replacing $\dot{\mathbf{A}}$ by $\mathbf{E}$ according to Eq. 7 the Hamiltonian can be expressed as

$$
H_{D}=\sum_{i}^{N} \frac{1}{2 m_{i}}\left(\mathbf{p}_{i}-q_{i} \mathbf{A}\right)^{2}+\frac{\Omega}{8 \pi} \mathbf{E}^{2}+V_{\mathrm{PBC}}\left(\mathbf{r}^{N}\right)-\Omega \mathbf{j}_{0} \cdot \mathbf{A}
$$

Eq. 23 has the same from as the minimal coupling Hamiltonian of electrodynamics (Recall however that the divergence and curl of all fields vanish).

\subsection{Transformation to Hamiltonian in $\mathrm{P} \cdot \mathrm{D}$ form}

The quantity $\mathbf{A}$ was introduced as a device to formulate a second order differential equation in time determining the time evolution of the uniform Maxwell field $\mathbf{E}$. 
Explicit dependence on A can be eliminated exploiting again the parallel to electrodynamics. This will also replace the coupling to $\mathbf{j}_{0}$ by a coupling to $\mathbf{D}$, its time integral (see Eq. 20). The idea is to transform the Lagrangian Eq. 10 by adding the total time derivative of a conveniently chosen function $F\left(\mathbf{r}^{N}, \mathbf{A}\right)[17]$

$$
\tilde{L}_{D}=L_{D}+\frac{d F}{d t}
$$

According to Hamilton's principle, the dynamics is not affected, only the canonical momenta change and therefore the Hamiltonian. The function we will use is

$$
F=\frac{\Omega}{4 \pi}(\mathbf{D}-4 \pi \mathbf{P}) \cdot \mathbf{A}
$$

Evaluating the time derivative

$$
\frac{d F}{d t}=-\Omega \mathbf{j}_{t} \cdot \mathbf{A}+\frac{\Omega}{4 \pi}(\mathbf{D}-4 \pi \mathbf{P}) \cdot \dot{\mathbf{A}}
$$

where we have used Eqs. 11, 3 and 20. Adding to $L_{D}$ according to Eq. 24 and substituting Eq. 10 we see that the current coupling term cancels

$$
\tilde{L}_{D}=\frac{1}{2} \sum_{i}^{N} m_{i} \mathbf{v}_{i}^{2}+\frac{\Omega}{8 \pi} \dot{\mathbf{A}}^{2}-V_{\mathrm{PBC}}\left(\mathbf{r}^{N}\right)+\frac{\Omega}{4 \pi}(\mathbf{D}-4 \pi \mathbf{P}) \cdot \dot{\mathbf{A}}
$$

Canonical momenta are back to the regular kinetic momenta

$$
\tilde{\mathbf{p}}_{i}=\frac{\partial \tilde{L}}{\partial \mathbf{v}_{i}}=m_{i} \mathbf{v}_{i}
$$

Now, however, the expression for the canonical momentum of $\mathbf{A}$ consists of multiple terms

$$
\tilde{\boldsymbol{\Pi}}_{A}=\frac{\partial \tilde{L}_{D}}{\partial \dot{\mathbf{A}}}=\frac{\Omega}{4 \pi}(-\mathbf{E}+\mathbf{D}-4 \pi \mathbf{P})
$$

where we have inserted Eq. 7 for $\dot{\mathbf{A}}$. It is easy to verify that the equations of motion remain the same. The forces on the particles have changed to

$$
\frac{\partial \tilde{L}_{D}}{\partial \mathbf{r}_{i}}=-\frac{\partial V_{\mathrm{PBC}}}{\partial \mathbf{r}_{i}}-\Omega \frac{\partial \mathbf{P}}{\partial \mathbf{r}_{i}} \cdot \dot{\mathbf{A}}
$$

The gradient of polarization is still simply the charge $q_{i} / \Omega$ times the unit matrix (Eq. 5) while $\dot{\mathbf{A}}$ is minus the electric field (Eq. 7). Together with Eq. 29 this indeed leads again to Eq. 15.

The equation of motion for $\mathbf{A}$ is found from Eq. 17 applied to the Lagrangian $\tilde{L}_{D}$. The force $\partial \tilde{L}_{D} / \partial \mathbf{A}$ on $\mathbf{A}$ vanishes, because the new Lagrangian is no longer an explicit function of $\mathbf{A}$. The conclusion is that the canonical momentum of $\mathbf{A}$ has been 
transformed in a constant of motion and thus according to Eq. 29

$$
\frac{d \tilde{\mathbf{\Pi}}_{A}}{d t}=\frac{\Omega}{4 \pi}(-\dot{\mathbf{E}}+\dot{\mathbf{D}}-4 \pi \dot{\mathbf{P}})=0
$$

Because of our definition Eq. 20 for the time derivative of $\mathbf{D}$, Eq. 31 is identical to the equation of motion Eq. 19. The dielectric relation Eq. 6 is satisfied at the microscopic level because it is invariant under the dynamics. Note also that this formal mechanical argument fails if we had immediately had set $\mathbf{D}-4 \pi \mathbf{P}$ in Eq. 27 to $\mathbf{E}=-\dot{\mathbf{A}}$.

The Hamiltonian corresponding to the Lagrangian Eq. 27 follows applying the general relation Eq. 21 using the transformed canonical momenta of Eqs. 28 and 29. The key term is the $\tilde{\boldsymbol{\Pi}} \cdot \dot{\mathbf{A}}$ product. Using Eqs. 29 and 7 we can write

$$
\tilde{\mathbf{\Pi}}_{A} \cdot \dot{\mathbf{A}}=\frac{\Omega}{4 \pi} \dot{\mathbf{A}}^{2}+\frac{\Omega}{4 \pi}(\mathbf{D}-4 \pi \mathbf{P}) \cdot \dot{\mathbf{A}}
$$

The second term in Eq. 32 fully cancels out the coupling term in the expression of Eq. 27 for $\tilde{L}_{D}$. What is left is only the kinetic term $\Omega \dot{\mathbf{A}}^{2} / 8 \pi$. Substituting $\dot{\mathbf{A}}=-\mathbf{E}=$ $4 \pi \mathbf{P}-\mathbf{D}$ we finally obtain

$$
\tilde{H}_{D}=\sum_{i}^{N} \frac{\mathbf{p}_{i}^{2}}{2 m_{i}}+\frac{\Omega}{8 \pi}(\mathbf{D}-4 \pi \mathbf{P})^{2}+V_{\mathrm{PBC}}\left(\mathbf{r}^{N}\right)
$$

Eq. 33 is the constant-D Hamiltonian of Eq. 2. Alternatively we could have held on to the canonical momentum $\tilde{\boldsymbol{\Pi}}_{A}$ instead of the time derivative $\dot{\mathbf{A}}$ finding the canonical expression for $\tilde{H}$,

$$
\tilde{H}_{D}=\sum_{i}^{N} \frac{\mathbf{p}_{i}^{2}}{2 m_{i}}+\frac{\Omega}{8 \pi}\left(\frac{4 \pi}{\Omega} \tilde{\mathbf{\Pi}}_{A}-(\mathbf{D}-4 \pi \mathbf{P})\right)^{2}+V_{\mathrm{PBC}}\left(\mathbf{r}^{N}\right)
$$

The $\tilde{\boldsymbol{\Pi}}_{A}$ term is of no consequence for the calculation of the energy. It is a constant of motion (Eq. 31) equal to zero assuming that this was the initial value. It is required however if one would wish to derive the first order Hamiltonian equations of motion.

\section{Constant-E}

How can we modify the derivation of section 2 to obtain the expression for the constant$\mathbf{E}$ Hamiltonian of Eq. 1? While $\mathbf{D}$ was fixed and $\mathbf{E}$ was a dynamical degree of freedom for constant-D, under constant-E conditions it is the other way around. This suggest introducing a "time-potential" $\mathbf{X}$ for $\mathbf{D}$ similar to $\mathbf{A}$ of Eq. 7 for $\mathbf{E}$.

$$
\dot{\mathbf{X}}=-\mathbf{D}
$$

$\mathbf{D}$ in turn was defined as the time derivative of the external current $\mathbf{j}_{0}$ in Eq. 20 which should be compared to the equivalent relation Eq. 18 for E. Under constant-E clearly $\dot{\mathbf{E}}=0$ which according to Eq. 19 implies that the fluctuations in the external current jo and polarization (internal current) cancel each other. However, there is no explicit external charge crossing the system. Not having to count ionic charge as external 
charge was what attracted us to the SSV approach in the first place. $\mathbf{j}_{0}$ must therefore be seen as a (transient) displacement current associated with the variation in time of the external charge on the virtual electrodes representing the electric boundary conditions in Ewald summation. After relaxing to zero, its time integral leaves a finite electric displacement $\mathbf{D}$.

Having decided for $\mathbf{X}$ of Eq. 35 as the dynamical degree of freedom for constant-E we have to specify a kinetic energy and a coupling term to the internal current. Rather than adapting the constant-D Lagrangian of section 2 we will postulate a Lagrangian $L_{E}$ and show that the chosen form leads to the correct equations of motion. The Lagrangian we will use is

$$
L_{E}=\frac{1}{2} \sum_{i}^{N} m_{i} \mathbf{v}_{i}^{2}+V_{\mathrm{PBC}}-\frac{\Omega}{8 \pi}(\dot{\mathbf{X}}+4 \pi \mathbf{P})^{2}
$$

with the polarization $\mathbf{P}$ again derived from the internal current $\mathbf{j}$ according to Eqs. 3 and 5. Expanding the square we see that Eq. 36 is of similar form as the transformed Lagrangian $\tilde{L}_{D}$ of Eq. 27 except for the alarming minus sign of the kinetic energy of $\mathbf{X}$. Such counter intuitive minus signs are characteristic of constant-E functionals. We will return to this issue in section 4 after investigating the equations of motion generated by Eq. 36 .

In common with Eq. 27 the Lagrangian of Eq. 36 is not explicitly dependent on currents and the canonical momentum of the particles is also equal to the kinetic momentum $\left(\mathbf{p}_{i}=m_{i} \mathbf{v}_{i}\right.$, see Eq. 28). The forces are more involved

$$
\begin{aligned}
\frac{\partial L_{E}}{\partial \mathbf{r}_{i}} & =-\frac{\partial V_{\mathrm{PBC}}}{\partial \mathbf{r}_{i}}-\Omega(\dot{\mathbf{X}}+4 \pi \mathbf{P}) \cdot \frac{\partial \mathbf{P}}{\partial \mathbf{r}_{i}} \\
& =-\frac{\partial V_{\mathrm{PBC}}}{\partial \mathbf{r}_{i}}+q_{i}(\mathbf{D}-4 \pi \mathbf{P})
\end{aligned}
$$

where we have used in the second equality Eq. 35. Substituting Eq. 6 we find Eq. 15 as required. However it still remains to be established that Eq. 6 in fact holds everywhere along a trajectory. This should follow from the equation of motion for $\mathbf{X}$ (or equivalently $\mathbf{D}$ ). We first obtain the canonical momentum conjugate to $\mathbf{X}$.

$$
\boldsymbol{\Pi}_{X}=\frac{\partial L_{E}}{\partial \dot{\mathbf{X}}}=-\frac{\Omega}{4 \pi}(\dot{\mathbf{X}}+4 \pi \mathbf{P})=\frac{\Omega}{4 \pi}(\mathbf{D}-4 \pi \mathbf{P})
$$

The forces on the field integral $\mathbf{X}$ vanish. $\boldsymbol{\Pi}_{X}$ of Eq. 38 is a constant of motion just as the momentum $\tilde{\boldsymbol{\Pi}}_{A}$ derived from the Lagrangian Eq. 27. The dynamics conserves the sum $\mathbf{D}-4 \pi \mathbf{P}$ allowing us to equate $\mathbf{D}-4 \pi \mathbf{P}$ with the constant Maxwell field $\mathbf{E}$. Moreover the momentum conjugate to $\mathbf{X}$ is also in effect equal to $\mathbf{E}$ (provided we keep volume fixed).

$$
\boldsymbol{\Pi}_{X}=\frac{\Omega}{4 \pi} \mathbf{E}
$$

This is the negative of Eq. 16, the momentum of $\mathbf{A}$ in the original current dependent minimal coupling Lagrangian of Eq. 10. Recall, however, that $\boldsymbol{\Pi}_{A}$ of Eq. 16 is not conserved. 
The expression for the Hamiltonian is found going through the regular steps. The all important term accounting for the dependence on the fields yields after substituting Eqs. 35 and 39

$$
\begin{aligned}
\boldsymbol{\Pi}_{X} \cdot \dot{\mathbf{X}}+\frac{\Omega}{8 \pi}(\dot{\mathbf{X}}+4 \pi \mathbf{P})^{2} & =-\frac{\Omega}{4 \pi} \mathbf{E} \cdot \mathbf{D}+\frac{\Omega}{8 \pi}(\mathbf{D}-4 \pi \mathbf{P})^{2} \\
& =-\frac{\Omega}{4 \pi} \mathbf{E} \cdot(\mathbf{E}+4 \pi \mathbf{P})+\frac{\Omega}{8 \pi} \mathbf{E}^{2} \\
& =-\frac{\Omega}{8 \pi} \mathbf{E}^{2}-\Omega \mathbf{E} \cdot \mathbf{P}
\end{aligned}
$$

where in the second equality we have substituted for D using Eq. 6. The resulting Hamiltonian is equal to Eq. 1 including the negative sign for the $\mathbf{E}^{2}$ term. Retaining the momentum we find the expression for the Hamiltonian in canonical form

$$
H_{E}=\sum_{i}^{N} \frac{\mathbf{p}_{i}^{2}}{2 m_{i}}-\frac{2 \pi}{\Omega} \boldsymbol{\Pi}_{X}^{2}-4 \pi \boldsymbol{\Pi}_{X} \cdot \mathbf{P}+V_{\mathrm{PBC}}\left(\mathbf{r}^{N}\right)
$$

Substituting Eq. 39 leads back to the Hamiltonian Eq. 1. However, the negative squared kinetic term is disconcerting. Fortunately, $H_{E}$ of Eq. 41 is invariant under changes in $\mathbf{X}$. The momentum $\boldsymbol{\Pi}_{X}$ is a constant of motion, avoiding mechanical instability. Even so, the mysterious minus sign is there for a purpose. It is needed to recover the correct Hamiltonian equations of motion for $\mathbf{X}$, as is verified below

$$
\dot{\mathbf{X}}=\frac{\partial H_{E}}{\partial \boldsymbol{\Pi}_{X}}=-\frac{4 \pi}{\Omega} \boldsymbol{\Pi}_{X}-4 \pi \mathbf{P}
$$

Inserting Eq. 39 we end up with Eq. 35 with the consistent sign.

\section{Summary and Outlook}

The constant-E Hamiltonian of Eq. 1 and constant-D Hamiltonian of Eq. 2 are a recent development in the Modern Theory of Polarization[29-32]. Eq. 3 is a central equation in this formalism leading to the famous Berry-phase expression for electronic polarization in periodic condensed phase systems. For classical systems straight forward integration of the internal current in Eq. 3 over time gives the dipole density expression of Eq. 5. For simple point charge (SPC) models of liquid water the polarization of Eq. 5 is rigorously equal to the sum of molecular dipoles divided by the volume of the MD cell. The Hamiltonians of Eqs. 1 and 2 can be directly applied in a finite field, constant volume calculation of the dielectric constant of liquid water[4, 13, 33]. Eq. 5 also holds for SPC models of ionic aqueous solution but with the subtlety that the dipole density must now be interpreted as itinerant polarization. This makes polarization multivalued similar to the Berry-phase expression for electronic polarization[30, 31]. However, when properly taken into account, Eqs. 1 and 2 can be equally used to simulate ionic solutions under finite field conditions [7, 34].

With the present contribution we hope to have given a clarification of the mechanical foundations of the finite field Hamiltonians $H_{E}$ of Eq. 1 and $H_{D}$ of Eq. 2. As a byproduct we obtained canonical expressions for these Hamiltonians, given in Eqs. 34 
and 41. The canonical Hamiltonians contain the conjugate momenta of the time integrals of the uniform fields which are the fundamental electric dynamical degrees of freedom in the extended Lagrangian. In practice explicit dependence on these conjugate momenta is however of no consequence for constant field molecular dynamics. Both quantities are constants of motion. As a result the fundamental relation of dielectrics (Eq. 6) is conserved by $H_{D}$ in its canonical form Eq. 34 as can be seen from Eq. 29. $H_{E}$ in canonical form Eq. 41 conserves the Maxwell field E. According to Eq. 39 the conjugate momentum of $\mathbf{X}$ is simply proportional to $\mathbf{E}$.

The minus $\mathbf{E}^{2}$ term in the electric enthalpy, the expectation value of $H_{E}$ of Eq. 1, has been a stumbling block in the development of variational Poisson Boltzmann methods $[35,36]$. The status of the uniform Maxwell field as a conserved dynamical variable in constant-E molecular dynamics adds a mechanical perspective to this term. A formal mechanical treatment, including canonical field momenta, could also be of interest in Liouville equation based time dependent perturbation theory[37]. Clearly there is a lot left to do in constant electric field molecular dynamics. There is already a static magnetic counter part of the modern theory of polarization(see the review by Resta[32]) which raises the question of coupling to magnetic fields. We also reiterate that all derivations in the paper assume that volume is held fixed. The constant pressure variant is a further interesting extension but likely not easy either[1, 14]. A firm mechanical foundation is hopefully of some help to meet these challenges.

\section{Acknowledgement}

Chao Zhang is acknowledged for the many discussions and insights about dielectrics in molecular dynamics.

\section{References}

[1] M. Stengel, N.A. Spaldin and D. Vanderbilt, Nat. Phys. 5 (4), 304-308 (2009).

[2] S.W. De Leeuw, J.W. Perram and E.R. Smith, Proc. Royal Soc. A 373, 27-56 (1980).

[3] J.M. Caillol, J. Chem. Phys. 101, 6080-6090 (1994).

[4] I.C. Yeh and M.L. Berkowitz, J. Chem. Phys. 110 (16), 7935-7942 (1999).

[5] L.L. Hirst, Rev. Mod. Phys. 69, 607-627 (1997).

[6] D.A. Kirzhnits, Sov. Phys. Usp. 30, 575-587 (1987).

[7] C. Zhang and M. Sprik, Phys. Rev. B 94, 245309 (2016).

[8] J.M. Caillol, D. Levesque and J.J. Weis, J. Chem. Phys. 85, 6645-6657 (1986).

[9] J.M. Caillol, D. Levesque and J.J. Weis, J. Chem. Phys. 91, 5544-5554 (1989).

[10] J.M. Caillol, D. Levesque and J.J. Weis, J. Chem. Phys. 91, 5555-5566 (1989).

[11] M. Sega, S.S. Kantorovich, A. Arnold and C. Holm, in Recent Advances in Broadband Dielectric Spectroscopy, edited by Yu P Kalmykov, NATO Science for Peace and Security Series B: Physics and Biophysic (Springer Science + Business Media, Dordrecht, 2013), pp. 103-122.

[12] M. Stengel, D. Vanderbilt and N.A. Spaldin, Phys. Rev. B 80, 224110 (2009).

[13] C. Zhang and M. Sprik, Phys. Rev. B 93, 144201 (2016).

[14] L.D. Landau and E.M. Lifschitz, Electrodynamics of continuous media. Course of theoretical physics volume 8 (Pergamon Press, Oxford, 1960).

[15] R.G. Woolley, Adv. Chem. Phys. 33, 153-233 (1975).

[16] R.G. Woolley, Int. J. Quantum Chem. 74, 531-545 (1999).

[17] E.A. Power and T. Thirunamachandran, Am. J. Phys. 46, 370- 378 (1978).

[18] W.P. Healy, Proc. Royal Soc. A 358, 367-383 (1977). 
[19] W.P. Healy, Phys. Rev. A 26, 1798-1799 (1982).

[20] M. Babiker and R. Loudon, Proc. Royal Soc. A 385, 439-460 (1983).

[21] V. Chernyak and S. Mukamel, Chem. Phys. 198, 133-143 (1995).

[22] E.A. Power and S. Zienau, Proc. Royal Soc. A 251, 427-454 (1959).

[23] J. Rottler and A.C. Maggs, Phys. Rev. Lett. 93, 170201 (2004).

[24] A.C. Maggs and R. Everaers, Phys. Rev. Lett. 96, 230603 (2006).

[25] V. Jadhao, F.J. Solis and M. Olvera de la Cruz, Phys. Rev. Lett. 109, 223905 (2012).

[26] F. Fahrenberger, Z. Xu and C. Holm, J. Chem. Phys. 141, 064902 (2014).

[27] A.C. Maggs, J. Chem. Phys. 120, 3108-3118 (2004).

[28] J. Schwinger, L.J. DeRaad Jr, K.A. Milton and W.Y. Tsai, Classical Electrodnamics (Advanced Book Program, Westview Press, Boulder, Colorado, 1998).

[29] R.D. King-Smith and D. Vanderbilt, Phys. Rev. B 47, 1651-1653 (1993).

[30] R. Resta, Rev. Mod. Phys. 66, 899-915 (1994).

[31] R. Resta and D. Vanderbilt, in Topics in Applied Physics Volume 105: Physics of Ferroelectrics: a Modern Perspective, edited by Karin M Rabe, Charles H Ahn and Jean-Marc Triscone (Springer-Verlag, Heidelberg, 2007), pp. 31-67.

[32] R. Resta, J. Phys.: Condens. Matter 22, 123201 (2010).

[33] C. Zhang, J. Hutter and M. Sprik, J. Phys. Chem. Lett. 7, 2696-2701 (2016).

[34] T. Sayer, C. Zhang and M. Sprik, J. Chem. Phys. 147, 104702 (2017).

[35] R. Allen, J.P. Hansen and S. Melchionna, Phys. Chem. Chem. Phys 3, 4177-4186 (2001).

[36] J.S. Pujos and A.C. Maggs, J. Chem. Theor. Comp. 11, 14191427 (2015).

[37] A. Nitzan, Chemical Dynamics in Condensed Phases Oxford graduate texts (Oxford University Press, Oxford, 2006). 